\section{How to form a fake memory in mice}

Unrelated memories can be artificially linked by activating distinct groups of neurons at the same time.

Kaoru Inokuchi at the University of Toyama, Japan, and his colleagues let mice explore a cylindrical container. Later they placed the mice in a cubic box and gave them an immediate electric shock to the foot. The scientists then used light to stimulate those neurons in the hippocampus and amygdala regions of the mouse brain that had been activated both when the animals had laid down their memories of the safe cylindrical container and when they received the electric shock in the box.

When light-stimulated mice were placed back in the cylinder, they froze for a longer time than mice that did not receive brain stimulation, suggesting that the stimulated mice connected the shock with the safe environment.

Cell Rep. http://dx.doi.org/10.1016/ j.celrep.2015.03.017 (2015)

\section{Wings that cope with collisions}

A wing inspired by those of birds and bats can overcome collisions with obstacles, and might allow the development of flying robots that have improved energy efficiency.

Amanda Stowers and David Lentink of Stanford University, California, built a robotic flapping wing consisting of an 'arm' attached to the body and a hinged 'hand' attached to that. The design naturally unfolds when the wing beats during flight. Modelling revealed that flapping generates acceleration, unfolding the wing.
The researchers showed in the lab that when the wing's hand hits a branch (pictured, left), it compensates by furling in (middle) and then unfurling (right), surviving impacts of up to 5 metres per second. This design could help future flapping robots to deal with tricky flight paths without the need for complicated algorithms, say the authors, because collisions could be survived rather than avoided. Bioinspir. Biomim. 10, 025001 (2015)

\section{GENOME EDITING}

\section{CRISPR controls gene expression}

A genome-editing technique can be modified to switch specific genes on and off through 'epigenetic' changes.

The technique, called CRISPR, allows biologists to edit selected DNA sequences, but it does not normally alter the epigenome - the chemical modifications to DNA and associated proteins that regulate gene expression. To target the epigenome, Timothy Reddy and Charles Gersbach at Duke University in Durham, North Carolina, and their colleagues mutated the enzyme used for CRISPR so that it no longer cut DNA, and fused it to part of an enzyme that transfers acetyl groups onto proteins associated with DNA.

The researchers targeted the modified enzyme to proteins near specific DNA sequences, and showed that the added acetyl groups boosted the expression of associated genes. The approach could be used to understand how specific epigenetic changes affect gene expression near the site of chemical modification and farther away, the authors say. Nature Biotechnol. http://dx.doi. org/10.1038/nbt.3199 (2015)
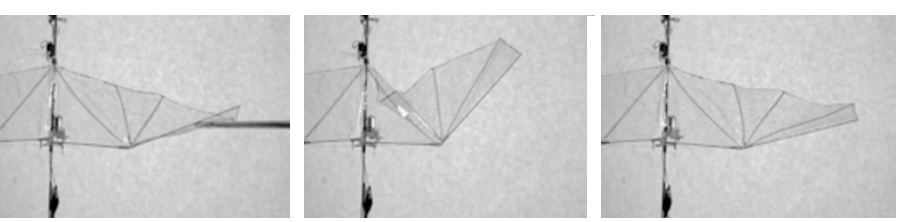

\section{Bacteria warm up permafrost}

Heat produced by microbes that break down organic material can speed up the thawing of frozen soils in the Arctic.

Thawing permafrost in a warming Arctic could release large amounts of organic carbon into the atmosphere, enhancing global warming. Bo Elberling at the University of Copenhagen and his colleagues determined the amount of heat produced by microbial activity in permafrost soils sampled from six sites in Greenland. They found that different types of organic soil are all susceptible to ground heat production, whereas mineral soils are less prone to it.

Using a model of heat and water flow, the scientists estimate that, as increasing temperatures begin to melt the permafrost, microbial heat production will also increase, accelerating the thaw.

Nature Clim. Change

Popular articles on social media the public

In a bid to make research papers more accessible to non-scientists, journals should require scientists to write lay summaries of their articles. That is the proposal put forward in a recent opinion piece that caught the attention of researchers on social media. Such summaries would clearly explain the importance and implications of the work, and drew support from some commenters online. "Lay summaries needed to enhance communication, visibility

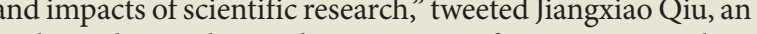
But Björn Brembs, a neuroscientist at the University of Regensburg in Germany, tweeted: "Such summaries sure are beneficial, but 'needed'? Written by the authors? Not so sure."

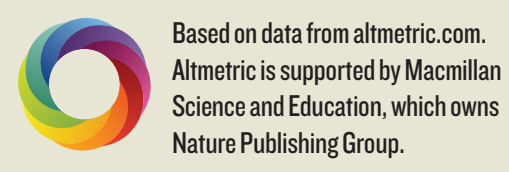

DNATURE.COM

For more on popular papers: go.nature.com/ti9wqn 